\title{
A Modified Neural Network Model for Lobula Giant Movement Detector with Additional Depth Movement Feature
}

\author{
Hongying Meng ${ }^{a}$ Shigang Yue ${ }^{a}$ Andrew Hunter $^{a}$ Kofi Appiah $^{a}$ \\ Mervyn Hobden ${ }^{b}$ Nigel Priestley ${ }^{b}$ Peter Hobden $^{b}$ Cy Pettit ${ }^{b}$
}

\begin{abstract}
The Lobula Giant Movement Detector (LGMD) is a wide-field visual neuron that is located in the Lobula layer of the Locust nervous system. The LGMD increases its firing rate in response to both the velocity of the approaching object and its proximity. It has been found that it can respond to looming stimuli very quickly and can trigger avoidance reactions whenever a rapidly approaching object is detected. It has been successfully applied in visual collision avoidance systems for vehicles and robots. This paper proposes a modified LGMD model that provides additional movement depth direction information. The proposed model retains the simplicity of the previous neural network model, adding only a few new cells. It has been tested on both simulated and recorded video data sets. The experimental results shows that the modified model can very efficiently provide stable information on the depth direction of movement.
\end{abstract}

\section{INTRODUCTION}

F Or animals, such as insects, the ability to detect approaching objects is important, serving both to prevent collision as the animal moves and also to avoid capture by predators [1][2]. Evolved over millions of years, the visual collision avoidance systems in insects are both efficient and reliable. The neural circuits processing visual information in insects are relatively simple compared to those in the human brain and provide a convenient model for the optical collision avoidance sensors that are needed to equip mobile intelligent machines [3].

The Lobula Giant Movement Detector (LGMD) is a widefield visual neuron that is located in the Lobula layer of the Locust nervous system. The LGMD increases its firing rate in response to both the velocity of the approaching object and its proximity. It has been found that it can respond to looming stimuli very quickly and can trigger avoidance reactions whenever a rapidly approaching object is detected. It is tightly tuned to respond to objects approaching the locust on a direct collision course [4], but produces little or no response to receding objects [5]. This makes the LGMD an ideal model to develop specialized sensors for automatic collision avoidance.

A functional neural network based on the LGMD's input circuitry was developed by Rind and Bramwell [6]. This four-layered neural network responds preferentially to approaching versus receding objects. It detects movement in depth by excitation, lateral inhibition and feed forward inhibition (FFI). Feed forward inhibition, which is sensitive

\footnotetext{
${ }^{a}$ Department of Computing and Informatics, University of Lincoln, UK (corresponding email: hmeng@lincoln.ac.uk)

${ }^{b}$ E2V Technologies PLC, Lincoln, UK.
}

to large background movements, is also believed to play a role in inhibiting LGMD spikes when presented with receding objects. This neural network has been used to mediate collision avoidance in a real-world environment by incorporating it into the control structure of a miniature mobile robot [7][8]. When integrated with robots, new cell feed-forward mediation (FFM), was introduced in [9][10] to mediate the response of the LGMD cell by varying its threshold to cope with extreme luminance conditions.

Given the inherent parallelism exhibited by this LGMD neural network model, it can be easily implemented in hardware, such as a Field Programmable Gate Array (FPGA) [11][12]. Laviana et al. [11][13] proposed a vision chip architecture that includes a block of $100 \times 150$ retinotopic units, a controller, a 16Kbits SRAM memory block, I/O registers and some other peripherals needed for addressing, timing control, digital-to analog converters and temperature monitoring. The LGMD neural network is designed on an FPGA platform and can deal with $100 \times 1506$-bit input image sequences. The chip has been designed in a $0.35 \mu \mathrm{m} 2 \mathrm{P}-2 \mathrm{M}$ technology. Okuno and Yagi [12] designed and implemented an LGMD model as a real-time vision sensor for collision avoidance. The system was confirmed to respond selectively to colliding objects even in complicated real-world situation.

Although the LGMD neural network model in [6] works very efficiently and is robust in many applications, particularly in collision avoidance, it does not provide significant information on the direction of depth movement. For example, the model may respond to both an approaching object and a receding object with equally high excitation levels, especially when an object is very close. The reason is that the difference between an approaching object and a receding object projected into FFI is often far less than the difference between background movements and an approaching object. It is hard for feed forward inhibition to play two roles well at the same time, even with fine tuning.

Inspired by the fact that there are direction selective neurons in the locust [14][15], A new specialized translationsensitive neural network (TSNN) has been proposed in [16][17]. The proposed TSNN neuron has some common layers with the LGMD model, allowing efficient implementation of the neural computations. The TSNN fuses extracted visual motion cues from several whole field direction selective neural networks, and is only sensitive to translational movements. It is unable to provide direction information on movements in depth. Given that the locust responds very strongly to approaching objects and responds very little to 
receding objects, we infer that the locust has the ability to reliably recognize the direction of the moving objects in depth. In order to enhance the ability to recognize the direction of the moving object in depth, we proposed a modified neural network model for LGMD with some extra cells, which provide the requisite direction information.

In comparison with the previous neural network models for LGMD [9], three more cells have been added in the proposed model. They are used to extract direction information on depth movement. Very little additional computing cost is required, and the proposed model has a negligible difference in efficiency compared to the previous models.

The rest of this paper is organized as follows. In section 2, we give a detailed description of the modified LGMD neural network model. In section 3, some experimental results on both the simulated and recorded video data set are shown; finally, we present conclusions.

\section{THE PROPOSED NEURAL NETWORK MODEL FOR LGMD}

The LGMD based neural network proposed in this paper is based on previous studies described in [6][8][18][9]. The modified neural network is shown in figure 1.The LGMD neural network in [6][8] was composed of four groups of cells - photoreceptor cells $(P)$; excitatory and inhibitory cells $(E$ and $I)$; summing cells $(S)$, and two single cells - feed-forward inhibition $(F F I)$ and LGMD. The neural network model of LGMD in [9] has an extra set of grouping cells between the summing cells and LGMD. This allows clusters of excitation in the summary cells to feed into the LGMD cell, which is useful for collision detection in complex backgrounds.

The input of the $P$ cells is the luminance change. Lateral inhibition is indicated with dotted lines and has a one frame delay. Excitation is indicated with black lines and has no delay. The $F F I$ also has a one frame delay. The input to $F F I$ is the luminance change from photoreceptor cells. The problem of parameter selection in this LGMD model has been tackled in [19].

The model in [9] works very well for collision detection in complex environments. However, it cannot distinguish the direction of moving objects in depth. For example, the model will respond to both an approaching object and a receding object with high excitation level, especially when an object is very close. In order to enhance the ability to recognize the direction of the moving object in depth, we add a new neural layer with two grouping cells $J$ and $H$ as shown in figure 1. A new cell $D$ is added to give the direction information of the moving object in depth. The proposed neural network (shown in figure 1) used in the paper will be described in detail in the next part (note that the $J, H$ and $D$ cells may not have exact counterparts in real locusts).

\section{A. P layer}

The first layer of the neural network is the photoreceptor $P$ cells arranged in a matrix; the luminance $L_{f}$ of each pixel in the input image is captured by each photoreceptor cell,

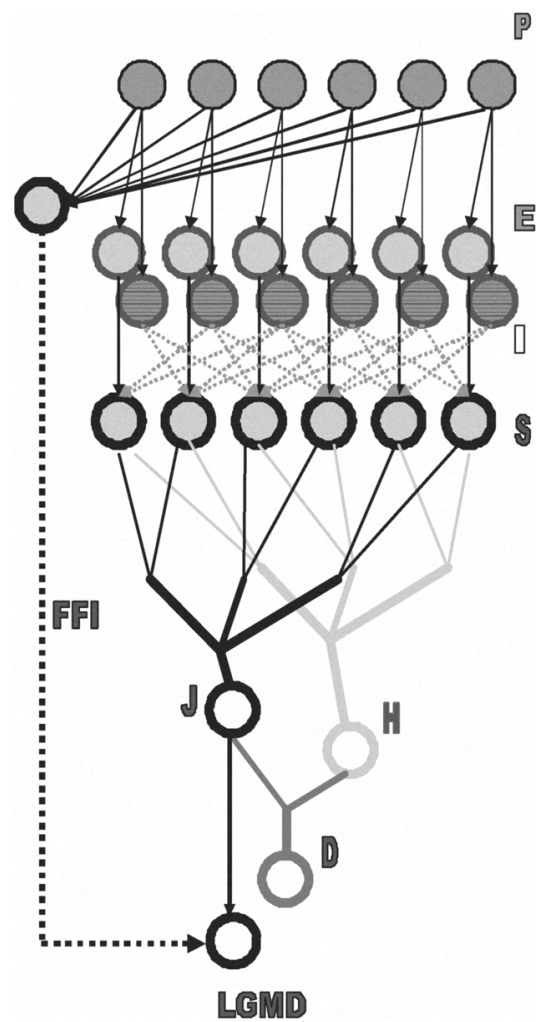

Fig. 1. A schematic illustration of the modified LGMD neural network model. There are four groups of cells and five single cells: photoreceptor cells (P); excitatory and inhibitory cells (E and I); summing cells (S); grouping cells $(\mathrm{J}$ and $\mathrm{H})$; depth movement direction cell (D); the LGMD cell and the feed forward inhibition cell (FFI).

the change of luminance $P_{f}$ between frames of the image sequence is calculated and forms the output of this layer. The output of a cell in this layer is defined by equation:

$$
P_{f}(x, y)=\sum_{i}^{n_{p}} p_{i} P_{f-i}(x, y)+\left(L_{f}(x, y)-L_{f-1}(x, y)\right)
$$

where $P_{f}(x, y)$ is the change of luminance corresponding to pixel $(x, y)$ at frame $f, x$ and $y$ are the indices of the matrix, $L_{f}$ and $L_{f-1}$ are the luminance, subscript $f$ denotes the current frame and $f-1$ denotes the previous frame, $n_{p}$ defines the maximum number of frames (or time steps) the persistence of the luminance change can last, the persistence coefficient $p_{i} \in(0,1)$ and

$$
p_{i}=\left(1+e^{\mu i}\right)^{-1}
$$

where $\mu \in(-\infty,+\infty)$ and $i$ indicates the previous $i^{t h}$ frame counted from the current frame $f$. Note that the LGMD neural network detects potential collisions by responding to expansion of the image edges, a strategy that needs computation rather than a strategy relying on object appearance. If there is no difference between successive images, the $P$ cells are not excited. 


\section{B. I E layer}

The output of the $P$ cells forms the inputs to two separate cell types in the next layer. One type are the excitatory cells, through which excitation is passed directly to their retinotopic counterparts in the third layer, the $S$ layer. The excitation $E(x, y)$ in an $E$ cell has the same value as that in the corresponding $P$ cell. The second cell type are lateral inhibition cells, which pass inhibition to their retinotopic counterpart's neighboring cells in the $S$ layer, with one frame delay. The gathered strength of inhibition of a cell in this layer is given by:

$$
I_{f}(x, y)=\sum_{i} \sum_{j} P_{f-1}(x+i, y+j) w_{I}(i, j), \quad(i=j \neq 0)
$$

where $I_{f}(x, y)$ is the inhibition corresponds to pixel $(x, y)$ at current frame $f, w_{I}(i, j)$ is the local inhibition weight. Note that $i$ and $j$ are not allowed to be equal to zero simultaneously. This means inhibition will only be allowed to spread out to its neighboring cells in next layer rather than to its direct counterpart in the next layer.

\section{S layer}

The excitatory flow from the $E$ cells and inhibition from the $I$ cells is summed by the $S$ cells using the following equation:

$$
S_{f}(x, y)=a b s\left(E_{f}(x, y)\right)-a b s\left(I_{f}(x, y)\right) W_{I}
$$

where $W_{I}$ is the inhibition weight. Excitations that exceed a threshold value are able to reach the summation cell LGMD:

$$
\tilde{S}_{f}(x, y)= \begin{cases}S_{f}(x, y), & \text { if } \quad S_{f}(x, y) \geq T_{r} \\ 0, & \text { if } \quad S_{f}(x, y)<T_{r}\end{cases}
$$

where $T_{r}$ is the threshold.

\section{J $H$ cells}

The $J$ and $H$ cells are the two new grouping cells for depth movement direction recognition. The $J$ cell is exactly the same as the LGMD cell in the previous LGMD model in terms of spatiotemporal structure and the value it holds. The $H$ cell shares the same structure as the LGMD and $J$ cell, but with a temporal difference. $H$ has one frame delay from $J$.

$$
\begin{gathered}
J_{f}=\sum_{x, y} \tilde{S}_{f}(x, y) \\
H_{f}=J_{f-1}
\end{gathered}
$$

\section{E. D cell}

The $D$ cell is used to calculate the difference between the difference of frame $f, f-1$ and $f-2$. It can be represented in the equation 8 .

$$
D_{f}=a b s\left(J_{f}\right)-a b s\left(H_{f}\right)
$$

It transpires that $D$ can estimate the depth direction of the moving object very well. When an object is moving away, $a b s\left(J_{f}\right)$ is always smaller than $a b s\left(H_{f}\right)$. When an object is approaching, $a b s\left(J_{f}\right)$ is bigger than $a b s\left(H_{f}\right)$. In order to distinguish slow movements we add a threshold $T_{D}$ for $D_{f}$. We then get a simple variable $\tilde{D}$ that has only three values, $0,1,-1$, where 1 stands for approaching, -1 stands for receding and 0 stands for no significant movements.

$$
\tilde{D}_{f}=\left\{\begin{array}{lll}
1, & \text { if } & D_{f} \geq T_{D} \\
0, & \text { if } & -T_{D}<D_{f}<T_{D} \\
-1, & \text { if } \quad D_{f} \leq-T_{D}
\end{array}\right.
$$

With the above cells, the modified neural model can recognise directional information for depth movements quickly. Feed forward inhibition cell, as detailed later, is able to concentrate on whole image movements to avoid perturbation from background movements.

\section{F. LGMD cell}

The membrane potential $J$ is then transformed to a spiking output using a sigmoid transformation,

$$
L G M D_{f}=\left(1+e^{-J_{f} n_{c e l l}^{-1}}\right)^{-1}
$$

where $n_{\text {cell }}$ is the total number of the cells in $S$ layer. Since $J_{f}$ is greater than or equal to zero (as equation 6 is a sum of absolute value), the sigmoid membrane potential $L G M D_{f}$ varies from 0.5 to 1 . The collision alarm is decided by the spiking of cell LGMD. If the membrane potential $L G M D_{f}$ exceeds the threshold $T s$, a spike is produced. A certain number of successive spikes, which is denoted by $S_{L G M D}$, will trigger the collision alarm in the LGMD cell. Of course, in the modified model, the collision alarm is only triggered under the condition that $\tilde{D}=1$ where the moving object is approaching. The spikes may be suppressed by the FFI cell when whole field movement occurs [18].

\section{G. FFI cell}

If it is not suppressed during turning, the network may produce spikes and even false collision alerts due to sudden changes in the visual scene. The feed forward inhibition and lateral inhibition work together to cope with such whole field movement [18]. The FFI excitation at the current frame is gathered from the photoreceptor cells with one frame delay,

$$
F_{f}=\sum_{j}^{n_{a}} \alpha_{f-j}^{F} F_{f-j}+\sum_{x=1}^{n_{r}} \sum_{y=1}^{n_{c}} a b s\left(P_{f-1}(x, y)\right) n_{c e l l}^{-1}
$$

where $\alpha_{f-j}^{F}$ is the persistence coefficient for $F F I$ and $\alpha_{f-j}^{F} \in(0,1), n_{a}$ defines how many time steps the persistence can last.

Once $F_{f}$ exceeds its threshold $T_{F F I}$, spikes in the LGMD are inhibited immediately. The threshold $T_{F F I}$ is also adaptable,

$$
T_{F F I}=T_{F O}+\alpha_{f f i} T_{F F I_{f-1}}
$$


where $T_{F O}$ is the initial value of the $T_{F F I}$, the adaptable threshold is decided by the previous $T_{F F I}$ and $\alpha_{f f i}$ is a coefficient.

As described in this section, the modified neural network model for LGMD only involves low level image processing, such as excitation transfer and neighborhood operations; computationally expensive methods, such as object recognition or scene analysis, are not used. Consequently, the proposed neural network model is able to work in real time and is independent of object classification.

\section{EXPERIMENTAL RESULTS ON THE PROPOSED MODEL}

Two different data sets were used to test the efficiency and stability of the proposed neural network model for LGMD. The first experiment is on a simulated data set that demonstrates approaching and receding movements. The second data sets are two recorded video clips. The parameters such as thresholds and inhibition weights were kept the same in all the experiments.

\section{A. Results on simulated data set}

We create a image sequence that contain 125 images with the approaching and receding movement of a square object. Some image frames are shown in figure 2 with the image size of $150 \times 100$. The square object with an initial size of $3 \times 3$ starts to approach from frame 5 to frame 41 and then recedes from frame 41 to frame 79 . The square object increases or decreases its border by 2 pixels (1 pixel on each side) per frame. From frame 79 to frame 84 , the square object is kept at same size of $3 \times 3$. Then the object approaches from frame 84 to frame 101 and then recedes from frame 101 to frame 120. This time, The square object increases or decreases its border by 4 pixels ( 2 pixel on each side) per frame. The square object is kept at same size of $3 \times 3$ from frame 120 to frame 125 .

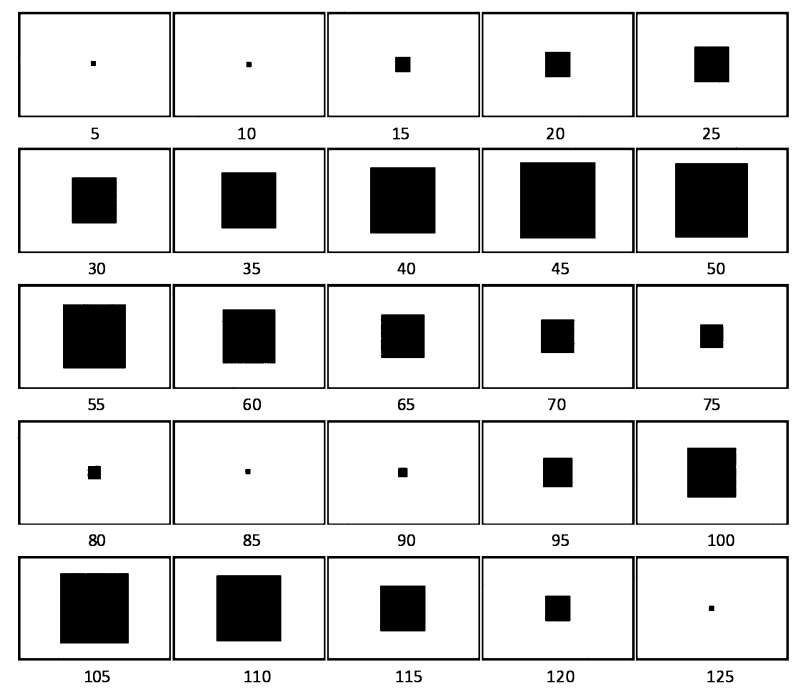

Fig. 2. The simulated image sequence generated in the experiment. There are 125 frames in all where an square object changes size from small $(3 \times 3)$ to big and then from big to small in the first part. A similar process occurs in the second part at higher speed.
Figure 3 shows the output of the modified modified LGMD neural network model on the simulated image sequence shown in figure 2. The vertical axis is the normalized membrane potentials of the LGMD cell. It is identical to the previous LGMD based neural network model. The other output of the proposed neural network is the direction of the depth movement, represented by the markers on the normalized membrane potentials of the LGMD cell; ' $\triangle$ ' indicates an approaching object, ' $\nabla$ ' a receding object and 'o' no significant movement. From figure 3 , we can clearly see that the output of the proposed LGMD neural network model works very well on the simulated data set.

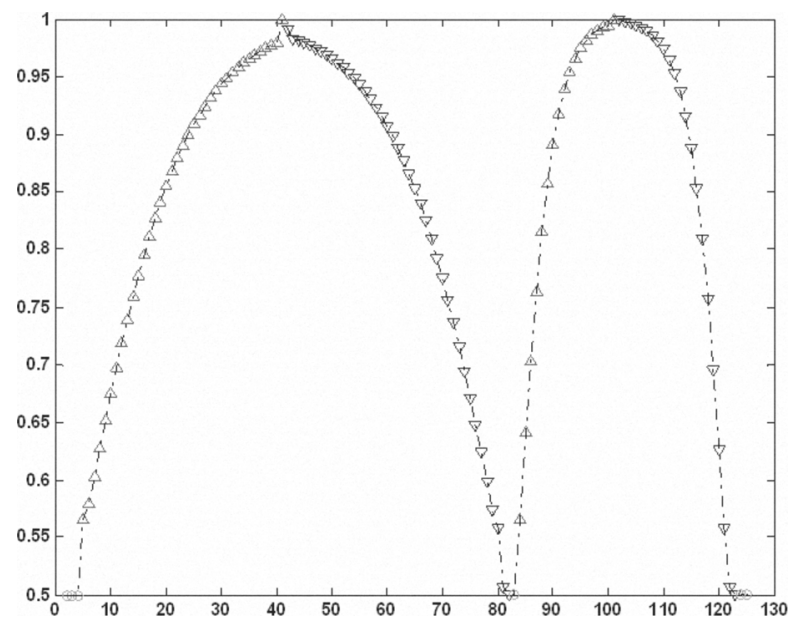

Fig. 3. The output of the modified LGMD based neural network model on the simulated image sequence shown in figure 2 . The vertical axis shows the normalized membrane potentials of the LGMD cell; the markers denote the depth movement direction of the object. ' $\triangle$ ' denotes an approaching; ' $\nabla$ ' a receding object and 'o' no significant movement.

\section{B. Results on real recorded data}

We recorded two short video clips (shown in figures 4 and 6 respectively) for the second experiment, using $320 \times 240$ gray scale images. In these two recorded videos (4), a ball is shown, mainly receding to the chair and then bouncing back to approach the camera. There are 18 and 21 frames in the first and second image sequences respectively. The first recording has a bigger, fast-moving ball while the second has a smaller, slower-moving ball.

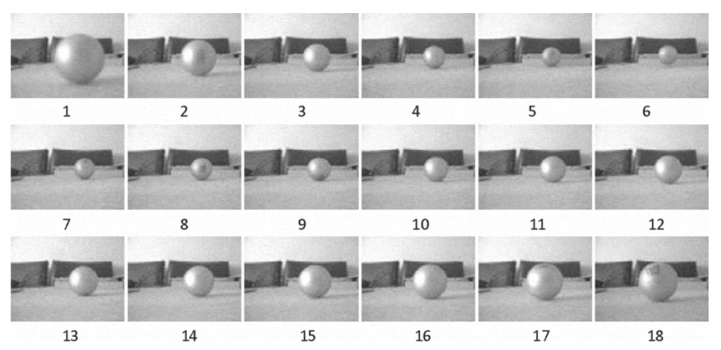

Fig. 4. The first recorded image sequence used in the experiment. There are 18 frames featuring a ball receding from the camera and then bouncing back to the camera after it hits a chair. 


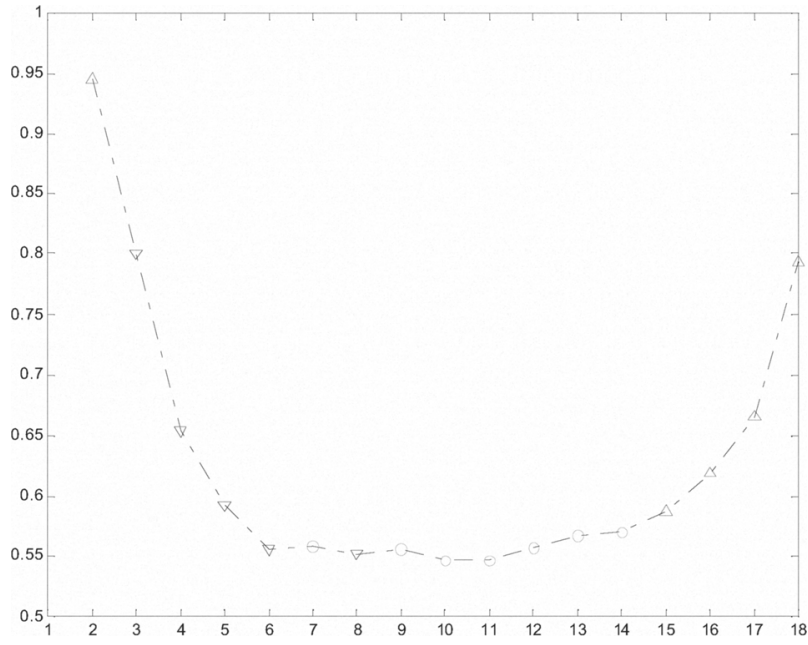

Fig. 5. The output of the modified LGMD based neural network model on the first recorded image sequence shown in figure 4. The vertical axis is the normalized membrane potentials of the LGMD cell. The markers denote the depth movement direction; ' $\triangle$ ' denote approaching objects; ' $\nabla$ ' receding objects and ' $o$ ' no significant movement.

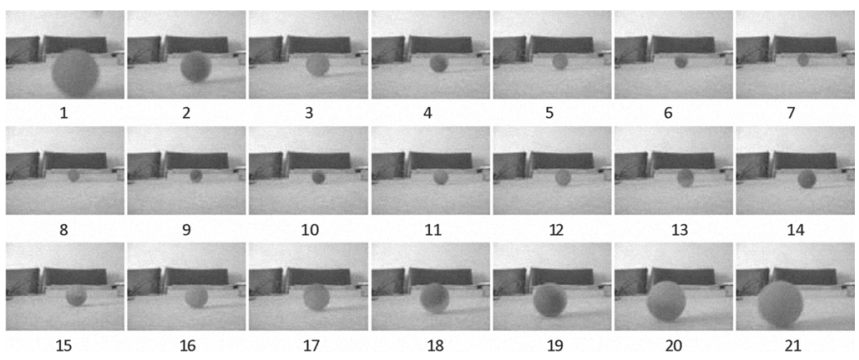

Fig. 6. The second recorded image sequence used in the experiment. There are 21 frames, featuring a ball receding from the camera and then bouncing back towards the camera after it hits the chair.

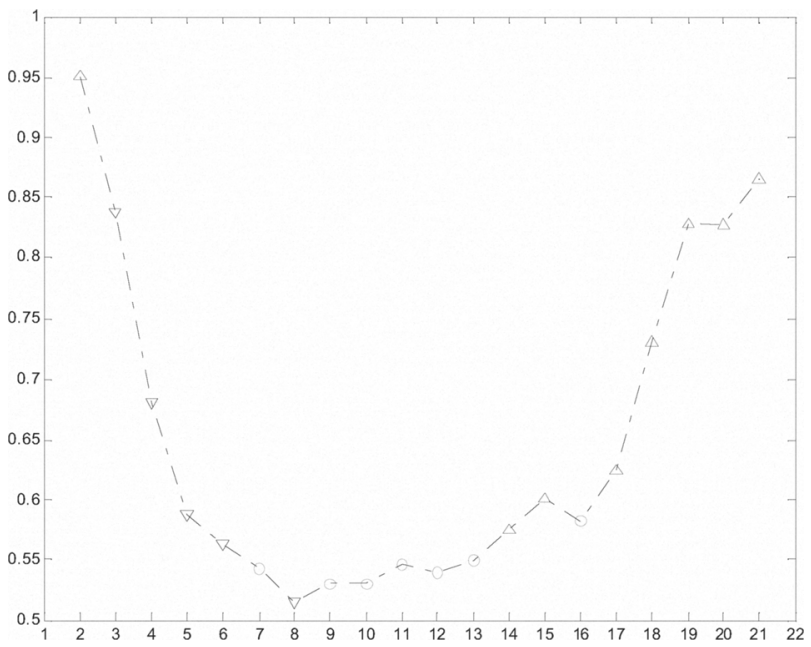

Fig. 7. The output of the modified LGMD based neural network model on the second recorded image sequence shown in figure 6 . The vertical axis is the normalized membrane potentials of the LGMD cell. The markers denote the depth movement direction; ' $\triangle$ ' denote approaching objects; ' $\nabla$ ' receding objects and ' $o$ ' no significant movement.
Figure 5 and 7 show the output of the modified modified LGMD based neural network model on the recorded image sequences shown in figure 4 and 6 respectively. The vertical axis gives the normalized membrane potentials of the LGMD cell. It is identical to the previous LGMD based neural network model. The other output of the proposed neural network is the direction of the depth movement, represented by the markers on the normalized membrane potentials of the LGMD cell; ' $\triangle$ ' denotes an approaching object; ' $\nabla$ ' a receding object and 'o' no significant movement. From figure 5 and 7, we can clearly see that the proposed LGMD neural network model works very well on both the two recorded data sets, although the speed of the movements are different in these two recordings.

\section{CONCLUSION AND DISCUSSION}

In this paper, we propose a modified neural network model for LGMD that provides additional information on the depth direction of the movement. The modified model is created and designed based on the previous LGMD models with little extra computational cost. It has some layers in common with the previous LGMD model, allowing efficient neural computation, and can recognize whether the object is approaching or receding very quickly. The modified model has been tested on both simulated and recorded video data sets. Experimental results showed the efficiency and stability of the proposed LGMD model.

In combination with the TSNN model [16], this modified LGMD neural network model has an enhanced ability to recognize the direction of the object movements. For applications such as collision detection for vehicles and robots, the model can remove the false collision alarm that happens in the previous model when a nearby object is moving away.

In this work, it is assumed that only one main object is moving in the scene at one moment. It is almost always true in the collision avoiding applications. However, for the case where there are more than one object while some objects approaching and some objects receding, the output of the $D$ cell(only one value) is not enough to give the direction information for all the depth movements. In this case, the main object needs to be focused and targeted locally using some low level image segmentation or localization techniques, then this model can provide the depth direction information for the main moving object. In future work, we will exploit the inherent parallelism of these LGMD neural network models to produce a complete FPGA design and implementation.

\section{REFERENCES}

[1] F. C. Rind and P. J. Simmons, "Seeing what is coming: building collision-sensitive neurones." Trends in Neurosciences, vol. 22, pp. 215-220, 1999.

[2] R. D. Santer, P. J. Simmons, and F. C. Rind, "Gliding behaviour elicited by lateral looming stimuli in flying locusts." Journal of comparative physiology. A, Neuroethology, sensory, neural, and behavioral physiology, vol. 191, no. 1, pp. 61-73, January 2005.

[3] F. C. Rind, R. D. Santer, M. J. Blanchard, and P. F. M. J. Verschure, Locust looming detectors for robot sensors. Berlin: Springer, 2003 2003, iD: 75; ID: 2921. 
[4] S. Judge and F. Rind, "The locust DCMD, a movement-detecting neurone tightly tuned to collision trajectories," Journal of Experimental Biology, vol. 200, no. 16, pp. 2209-2216, 1997. [Online]. Available: http://jeb.biologists.org/cgi/content/abstract/200/16/2209

[5] F. C. Rind and P. J. Simmons, "Orthopteran demd neuron: a reevaluation of responses to moving objects. i. selective responses to approaching objects," $J$ Neurophysiol, vol. 68, no. 5, pp. 1654-1666, 1992. [Online]. Available: http://jn.physiology.org/cgi/content/abstract/68/5/1654

[6] F. Rind and D. Bramwell, "Neural network based on the input organization of an identified neuron signaling impending collision." Journal of Neurophysiology, vol. 75, pp. 967-985, 1996.

[7] M. J. Blanchard, F. C. Rind, and P. F. M. J. Verschure, "Using a mobile robot to study locust collision avoidance responses," International journal of neural systems, vol. 9, pp. 405 - 410, 1999 1999, iD: 53; ID: 601 .

[8] M. Blanchard, F. Rind, and P. Verschure, "Collision avoidance using a model of the locust lgmd neuron." Robotics and Automonous Systems, vol. 30 , pp. $17-38,2000$.

[9] S. Yue and F. C. Rind, "A collision detection system for a mobile robot inspired by the locust visual system," in ICRA, 2005, pp. 3832-3837.

[10] - "Collision detection in complex dynamic scenes using an lgmd-based visual neural network with feature enhancement," IEEE Transactions on Neural Networks, vol. 17, no. 3, pp. 705-716, 2006.

[11] R. Laviana, L. Carranza, S. Vargas, G. Linan, and E. Roca, "A bioinspired vision chip architecture for collision detection in automotive applications," in Society of Photo-Optical Instrumentation Engineers (SPIE) Conference Series, ser. Society of Photo-Optical Instrumentation Engineers (SPIE) Conference Series, R. A. Carmona and G. Linan-Cembrano, Eds., vol. 5839, Jun. 2005, pp. 13-24.

[12] H. Okuno and T. Yagi, "Real-time robot vision for collision avoidance inspired by neuronal circuits of insects," in IROS, 2007, pp. 13021307.

[13] J. Cuadri, G. Linan, R. Stafford, M. S. Keil, and E. Roca, "A bioinspired collision detection algorithm for vlsi implementation," R. A. Carmona and G. Linan-Cembrano, Eds., vol. 5839, no. 1. SPIE, 2005, pp. 238-248. [Online]. Available: http://link.aip.org/link/?PSI/5839/238/1

[14] F. C. Rind, "A directionally selective motion-detecting neurone in the brain of the locust: physiological and morphological characterization," Journal of Experimental Biology, vol. 149, pp. 1-19, 1990.

[15] — , "Identification of directionally selective motion-detecting neurones in the locust lobula and their synaptic connections with an identified descending neurone," Journal of Experimental Biology, vol. 149, pp. 21-43, 1990.

[16] S. Yue and F. C. Rind, "Visual motion pattern extraction and fusion for collision detection in complex dynamic scenes," Computer Vision and Image Understanding, vol. 104, no. 1, pp. 48 - 60, 2006. [Online]. Available: http://www.sciencedirect.com/science/article/B6WCX4KPNB5D-1/2/441b9f636b155af920180f6391 ca0fc1

[17] _ _ "A synthetic vision system using directionally selective motion detectors to recognize collision," Artificial Life, vol. 13, no. 2, pp. 93-122, 2007.

[18] R. D. Santer, R. Stafford, and F. C. Rind, "Retinally-generated saccadic suppression of a locust looming detector neuron: Investigations using a robot locust." Journal of the Royal Society: Interface, vol. 1, pp. 61-77, 2004.

[19] S. Yue, F. C. Rind, M. S. Keil, J. Cuadri, and R. Stafford, "A bioinspired visual collision detection mechanism for cars: Optimisation of a model of a locust neuron to a novel environment," Neurocomputing, vol. 69 , no. $13-15$, pp. 1591-1598, 2006. 